\title{
Simulation of Diblock Copolymer Self-Assembly, Using a Coarse-Grain Model
}

\author{
Goundla Srinivas, $, *,+$, John C. Shelley, ${ }^{\S}$ Steve O. Nielsen, ${ }^{\dagger}$ Dennis E. Discher,${ }^{\perp}$ and \\ Michael L. Klein \\ Center for Molecular Modeling, Department of Chemistry, University of Pennsylvania, \\ Philadelphia, Pennsylvania 19104-6323, Schroedinger, Inc., 1500 S.W. First Avenue, Suite 1180, \\ Portland, Oregon 97201, Department of Chemical \& Biomolecular Engineering, University of Pennsylvania, \\ Philadelphia, Pennsylvania 19104-6315, and Laboratory for Research on the Structure of Matter, \\ University of Pennsylvania, Philadelphia, Pennsylvania 19104-6202
}

Received: December 12, 2003; In Final Form: April 12, 2004

\begin{abstract}
A coarse-grain model for amphiphilic diblock copolymers is developed by fitting the required parameters to properties taken from all-atom molecular dynamics simulations and experimental data. Computations with the present coarse-grain model yield spontaneous self-assembly of a random system into membrane bilayers when the amphiphilic diblock copolymers have a lipid-like hydrophilic/hydrophobic ratio. The model semiquantitatively reproduces a number of experimental results that were not explicitly used in the parametrization. In particular, diblock polymers with the appropriate ratio of hydrophobic-hydrophilic segment lengths self-assemble into membranes whose hydrophobic thickness (determined from mass density profiles) and scaling with molecular weight are found to be in good agreement with the experiment.
\end{abstract}

\section{Introduction}

Natural lipids spontaneously form vesicles in aqueous media. ${ }^{1}$ Both static and dynamic properties of these membranes have been extensively studied over the years and well-characterized. ${ }^{2-8}$ However, defects and poor stability limit the utility of lipid vesicles in, for example, drug delivery ${ }^{9}$ and biosensor applications. Desirable membrane properties such as controlled degradation are also not so easily designed into vesicles of natural lipids. Structurally, all-natural lipid membranes have a narrow range of hydrophobic core thickness (typically 3-4 nm), making it difficult to probe membrane properties as a function of thickness. Any dramatic increase in core thickness would alter the hydrophobic/hydrophilic ratio, and thereby promote a phase transition to an inverted phase.

Such limitations with lipids have motivated development of nonnatural vesicles. ${ }^{10}$ Synthetic membrane components have included amphiphilic dendrimers, ${ }^{11}$ fullerene-based surfactants, ${ }^{12}$ and charge-balancing mixtures of cationic and anionic surfactants. ${ }^{13}$ Extensive studies have also shown that vesicles can be formed from organic super amphiphiles such as diblock copolymers. ${ }^{14-22}$ These can have similar hydrophobic/hydrophilic proportions to natural lipids, but consist of two distinct polymer chains covalently linked in series. ${ }^{23,24}$ Importantly, copolymer molecular weights can be considerably larger (3$20 \mathrm{kDa})$ than those of natural lipid membranes $(<1 \mathrm{kDa}){ }^{25}$ Although the synthesis and study of a wide range of block copolymers have been conducted, ${ }^{26}$ only recently have stable and well-characterized polymer vesicles or polymersomes been reported. ${ }^{15,17}$ One key example is a nonionic diblock copolymer

\footnotetext{
* Address correspondence to this author. E-mail: srini@ cmm.upenn.edu.

Center for Molecular Modeling, Department of Chemistry, University of Pennsylvania.

Laboratory for Research on the Structure of Matter, University of Pennsylvania.

$\S$ Schroedinger, Inc.

${ }^{\perp}$ Department of Chemical \& Biomolecular Engineering, University of Pennsylvania
}

first reported by Hillmayer and Bates, ${ }^{14}$ poly(ethylene oxide)polyethylethylene $\left(\mathrm{EO}_{40} \mathrm{EE}_{37}\right.$, designated as $\left.\mathrm{OE7}\right)$, that was subsequently demonstrated to self-assemble into unilamellar giant vesicles (termed polymersomes in analogy with liposomes) in a variety of purely aqueous conditions. Together with a range of related copolymers that also assembled into vesicles, the hydrophobic core thickness of this class of polymer membranes varies with copolymer molecular weight and in a range from $\sim 8-22 \mathrm{~nm} .{ }^{27}$ Hence, polymersomes provide a novel, experimental opportunity to study thickness dependence of membrane properties in a systematic way. In practical terms, many achievements with liposomes such as protein integration, fusion, DNA encapsulation, compatibility, etc. have been achieved with synthetic polymer vesicles, ${ }^{27}$ broadening the range of vesicle properties through a wide choice of amphiphile molecular weight, hydrophobic/hydrophilic fraction, and chemistry.

Polymersomes have thus proven to have interesting and useful properties. They are stable well in excess of biologically relevant temperatures ${ }^{27}$ and they exhibit remarkable mechanical stability; OE7 vesicles, for example, sustain $>20 \%$ mechanical dilation compared to phospholipid membranes that rupture at $<5 \%$ stretching. ${ }^{28,29}$ As suggested above, such low stability is attributed to smaller hydrophobic thickness, which makes lipid membranes more susceptible to fluctuations and defects. However, polymersome membranes have a lower lateral diffusivity and a higher viscosity, with a fluidity that decreases with increasing molecular weight and most dramatically with chain entanglement. ${ }^{17}$ Such properties have motivated the present simulation study, which is aimed at generating a better understanding of polymersomes from a microscopic viewpoint.

Block copolymer membranes raise a number of further questions that simulation can lend insight into. First, since all synthetic polymers are significantly polydisperse (OE7 is about 10 times more than natural membranes), ${ }^{27}$ simulated models can address the need ${ }^{30}$ or impact of polydispersity on vesicle formation. Second, a unifying feature of polymersomes is the 
phospholipid-like hydrophilic ratio $\left(f_{\text {hydrophilic }} \approx 35 \% \pm 10 \%\right)^{31}$ that is needed. Molecules with $f_{\text {hydrophilic }}<25 \%$ are expected to form inverted microstructures, reflective of a time-average shape being an inverted cone, while the molecules with $f_{\text {hydrophilic }}$ $>45 \%$ are expected to form micelles, ${ }^{27}$ reflective of a timeaverage shape being cone shaped.

Despite progress on the experimental and theoretical studies $^{32,33}$ of polymersomes no simulation studies have been reported. Thus many microscopic aspects of these fascinating systems remain to be understood. The unusually large system sizes (typically consisting of $>100000$ atoms) make the task of carrying out simulations computationally very demanding. Although the advent of PC clusters allows one to handle such systems, it is still impractical to routinely study phenomena such as the self-assembly of such large and slowly relaxing systems, which occurs typically on a multinanosecond to microsecond time scale. Existing simulation studies of block copolymers have been mostly carried out by studying model block copolymers with simplified interaction potentials. For example, dissipative particle dynamics (DPD), ${ }^{34-38}$ discontinuous molecular dynamics (DMD),${ }^{39}$ Monte Carlo simulations, ${ }^{40}$ and Brownian dynamics simulations ${ }^{41,42}$ have been used to study self-assembly of block copolymers by microphase separation. Lipowsky and coworkers $^{43,44}$ developed simplified models for solvent and amphiphiles and studied the self-assembled membrane properties by carrying out extensive simulations. Recently Klein and coworkers ${ }^{45-47}$ developed a coarse-grain (CG) model for phospholipid molecules that was found to be effective in studying various membrane properties. Marrink and co-workers ${ }^{48,49}$ demonstrated the efficiency of coarse-grain models by studying a variety of membrane phenomena such as membrane fusion and vesicle fusion. ${ }^{48}$ Marrink and Mark recently presented a new coarse-grained model for lipid and surfactant systems. ${ }^{50}$ In all these studies, the basic coarse-grain approach builds on pioneering works of Smit and co-workers. ${ }^{51,52}$ Encouraged by such studies, in this work we have developed a similar CG model for the diblock super amphiphiles mentioned above. Anticipating our results, we will show that our CG model is able to reproduce key experimental quantities reasonably well, including the spontaneous self-assembly of diblock copolymers with a lipid-like hydrophobic/hydrophilic ratio into membrane bilayer structures.

This paper is organized as follows. A detailed description of the CG model is presented in the next section. Section 3 contains details of the simulations on membranes composed of monodisperse OE7 polymers. The simulation results are presented in Section 4. The article ends with some remarks about future directions.

\section{The Coarse-Grain Model}

To develop the parameters of the CG model we need target observables, and these are taken either from experiments or from all-atom simulations. To this end, bulk density and surface tension values are taken from experimental studies, ${ }^{53,54}$ while the remaining quantities are obtained by carrying all-atom (AA) simulations. As already mentioned, all-atom simulations of these systems are computationally demanding. For example, a modest system containing $50 \mathrm{OE} 7$ polymer chains, with sufficient water to fully hydrate them, typically contains $100000-125000$ atoms. Instead of carrying out simulations with long $\mathrm{EO}_{m} \mathrm{EE}_{n}$ copolymers, we have employed an AA approach (based on the OPLS force field $)^{55,56}$ on minipolymer chains, as mimics of OE7 copolymers. These smaller chains are selected in a way to represent all the different segments present in the diblock
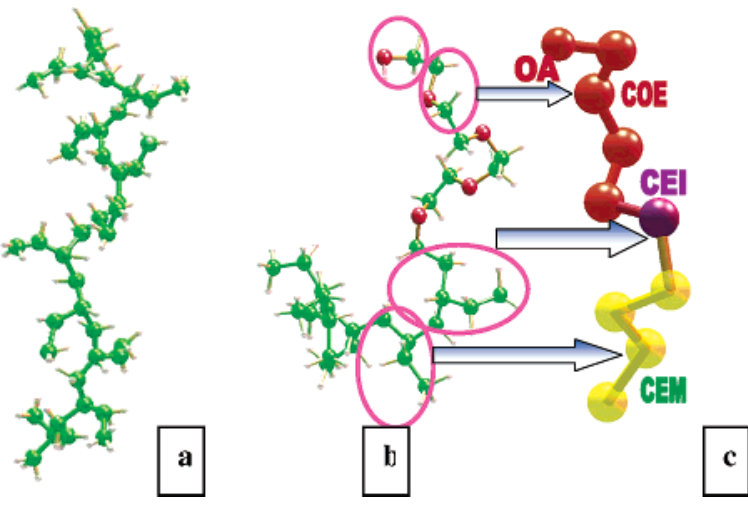

Figure 1. Representative polymer chains (a) PEE and (b) PEE-PEO studied by all-atom simulations. The scheme for mapping an all-atom diblock copolymer onto a coarse-grain model is also shown $(b \rightarrow c)$. The monomer units EO and EE are represented by the coarse-grain units COE and CEM, respectively. CEI denotes the interfacial unit, while the end groups, namely tertiary butyl and $\mathrm{CH}_{2}-\mathrm{OH}$, are represented by CET and OA, respectively. The color code for the allatom representation follows: carbon, green; hydrogen, white; and oxygen, red.

copolymer of interest. Figure 1a represents the hydrophobic (PEE) part of the diblock while Figure $1 \mathrm{~b}$ represents a copolymer consisting of hydrophilic and hydrophobic parts along with the interface region. Mapping of the all-atom (AA) diblock copolymer to coarse-grain units is pictorially shown in the same figure $(\mathrm{b} \rightarrow \mathrm{c})$. In this representation, each monomer constituting the polymer is represented by a single spherical $\mathrm{CG}$ unit. Thus, the ethylethylene monomer $-\mathrm{CH}_{2}-\mathrm{CH}\left(\mathrm{CH}_{2}-\right.$ $\mathrm{CH}_{3}$ ) - is represented by a single CEM unit while $-\mathrm{CH}_{2}-\mathrm{O}-$ $\mathrm{CH}_{2}-$ is represented by a single $\mathrm{COE}$ unit. The latter choice is preferred over an ethylene oxide monomer unit $-\mathrm{CH}_{2}-\mathrm{CH}_{2}-$ $\mathrm{O}-$ merely for symmetry reasons. CEI represents $-\mathrm{CH}_{2}-\mathrm{CH}-$ $\left(\mathrm{CH}_{2}-\mathrm{CH}_{3}\right)-\mathrm{CH}_{2}-$, a relatively larger group, present at the diblock interface. The tertiary butyl group present at the end of the ethylethylene block is represented by CET. Each of the polymer chains described above contains $10 \mathrm{CG}$ units. Note that in the CG approach the number of interacting sites is typically reduced by an order of magnitude.

As noted in previous studies, ${ }^{45,46}$ by grouping more than 3 $-\mathrm{CH}_{2}$ - groups into a single entity might result in the loss of structural information. However, in the present case we observe that due to the presence of the ethylene branch, grouping four carbons into a single spherical unit is a better approximation than grouping three linear $-\mathrm{CH}_{2}$ - groups into a spherical unit. In addition, this obviously results in simplified structure while retaining the basic topology of the polymer. In the following section we describe the CG parametrization in detail.

2.1. Hydrophobic PEE. An all-atom simulation with 144 PEE chains (as shown in Figure 1a) has been carried out for $0.5 \mathrm{~ns}$ in an NPT ensemble at $298.15 \mathrm{~K}$ and $1 \mathrm{~atm}$ of pressure with a 1 fs time step. Data collected during the simulation are utilized to generate bond distance and bond angle distributions for the consecutive monomers in the polymer chain. These results are used as target observables for CG parametrization as described below.

The CG polymer chain is constructed by using the centerof-mass position of each monomer of the AA polymer chain. Consecutive monomers in a polymer are bonded by harmonic potentials of the form,

$$
U_{\text {bond }}\left(r_{i j}\right)=\left(k_{\mathrm{b}} / 2\right)\left(r_{i j}-r_{\mathrm{o}}\right)^{2}
$$

Here the equilibrium bond distance is denoted by $r_{\mathrm{o}}$, and $k_{\mathrm{b}}$ is 

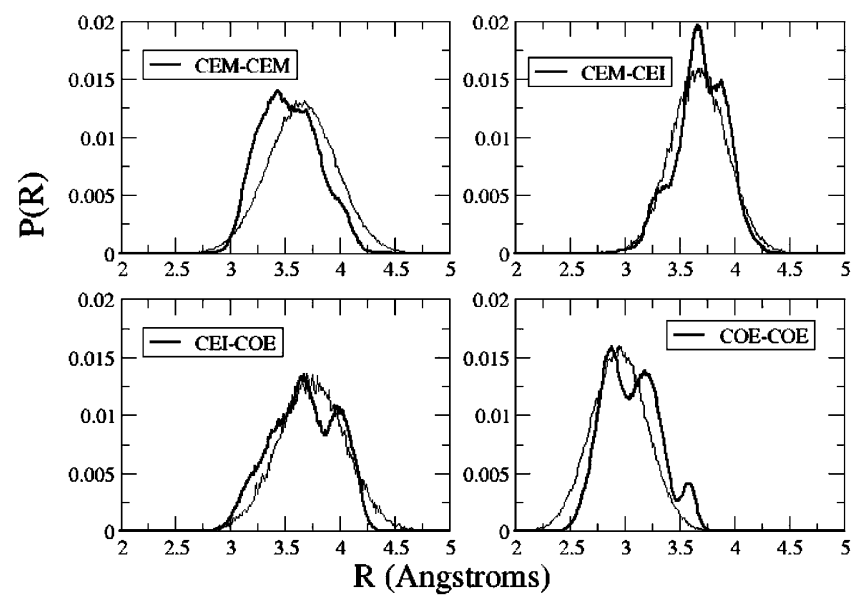

Figure 2. Comparison of group-group bond distributions obtained from AA (thick line) and CG (thin line) molecular dynamics simulations, respectively. The specific distributions are mentioned in each panel.

TABLE 1: Average Bond Lengths Obtained from All-Atom (AA) Simulations, and the Coarse-Grain Bond Potential Parameters for All the Monomers Present in $\mathbf{E O}_{m} \mathbf{E E}_{n}$ Copolymers

\begin{tabular}{lccc}
\hline \multicolumn{1}{c}{ bond } & $\begin{array}{c}\text { all-atom } \\
\langle r\rangle(\AA)\end{array}$ & $\begin{array}{c}\text { coarse-grain } \\
\langle r\rangle(\AA)\end{array}$ & $\begin{array}{c}\text { coarse-grain } \\
k_{\mathrm{b}}(\mathrm{K})\end{array}$ \\
\hline CET-CEM & 3.39 & 3.41 & 12400 \\
CEM-CEM & 3.70 & 3.86 & 3200 \\
CEM-CEI & 3.69 & 3.72 & 4850 \\
CEI-COE & 3.73 & 3.75 & 3250 \\
COE-COE & 2.97 & 2.97 & 4550 \\
COE-OA & 2.46 & 2.46 & 2815
\end{tabular}

the bond stretching force constant. The parameters $r_{\mathrm{o}}$ and $k_{\mathrm{b}}$ are determined by adjusting initial guesses until they satisfactorily reproduce the mean and variance of the bond distribution obtained from the AA simulation of the hydrophobic chain. Comparison between bond distributions obtained from AA and CG simulations is shown in Figure 2. The bond distances determined both from CG and AA are listed in Table 1. By following Lipowsky et al., ${ }^{43,44}$ the bond angle between three consecutive monomers in the polymer chain is determined by the cosine angle potential,

$$
U_{\text {bend }}\left(\theta_{i j k}\right)=k_{\theta}\left[1-\cos \left(\pi-\theta_{i j k}\right)\right]
$$

where $\theta_{i j k}$ is the bond angle formed by sites $i, j$, and $k$. The corresponding bond angle force constant $k_{\theta}$ was adjusted until the MD simulations reproduce atomistic bond angle distribution satisfactorily. Comparison between AA and CG bond angle distributions is shown in Figure 3. The bond-angle values obtained from both CG and AA are listed in Table 2.

Since the parametrization of the CG intramolecular model of the PEE chain is completed, we turn our attention to intermolecular interactions. In the $\mathrm{CG}$ representation, the interacting sites are physically larger than those of the AA model and hence we need potentials with wider wells. Accordingly, the nonbonded interactions among hydrophobic sites are modeled with a potential of the Lennard-Jones (9-6) form,

$$
U\left(r_{i j}\right)=(15 / 4) \epsilon\left[\left(\sigma / r_{i j}\right)^{9}-\left(\sigma / r_{i j}\right)^{6}\right]
$$

Here, $\epsilon$ and $\sigma$ correspond to the well-depth and van der Waals diameter of the corresponding species. To determine these parameters, we have chosen the experimental bulk density and surface tension as target observables (adopted from literature). ${ }^{53}$

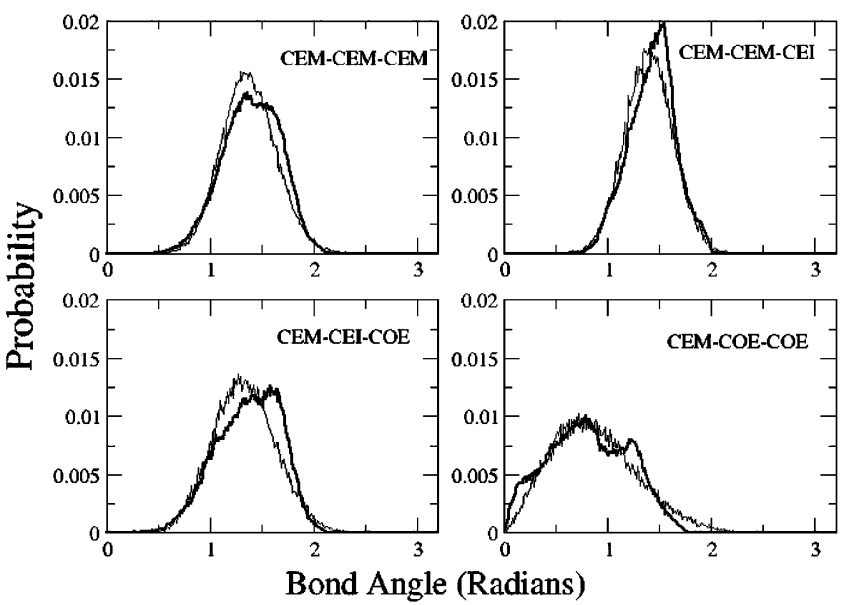

Figure 3. Comparison of bond angle distribution obtained from AA (thick line) and CG (thin line) MD simulations. The CG units involved in defining the bond angle are mentioned in the figure. As shown, by adopting the CG method the finer structural details are averaged out but the resulting distribution shows the correct overall behavior.

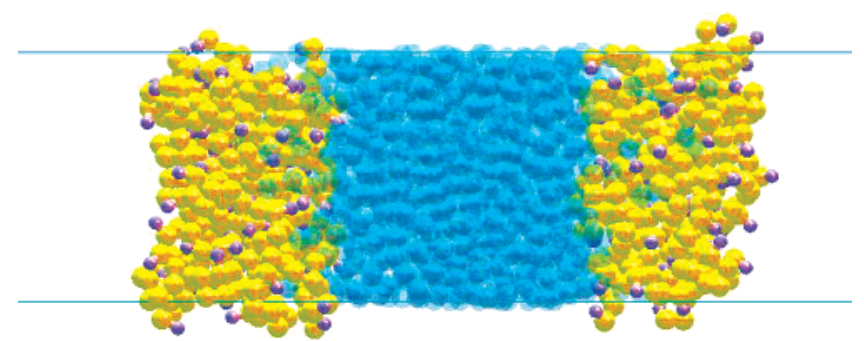

Figure 4. Simulation cell setup $(y-z$ plane) used to calculate the hydrocarbon-water interfacial tension as viewed along the $x$-axis cell. The color scheme is as follows: water, blue; hydrocarbon chains, yellow; and the hydrocarbon chain ends, violet.

TABLE 2: Comparison of Various Bond Angle Parameters Obtained from the AA and CG Models

\begin{tabular}{lccr}
\hline \multicolumn{1}{c}{ angle } & $\begin{array}{c}\text { all-atom } \\
\langle\theta\rangle(\mathrm{rad})\end{array}$ & $\begin{array}{c}\text { coarse-grain } \\
\langle\theta\rangle(\mathrm{rad})\end{array}$ & $\begin{array}{c}k_{\theta} \\
(\mathrm{K})\end{array}$ \\
\hline CET-CEM-CEM & 96.12 & 96.00 & 12400 \\
CEM-CEM-CEM & 102.3 & 102.0 & 5200 \\
CEM-CEM-CEI & 98.72 & 99.00 & 5600 \\
CEM-CEI-COE & 99.01 & 92.125 & 3300 \\
COI-COE-COE & 146.7 & 146.1 & 900 \\
COE-COE-COE & 138.1 & 138.1 & 1000 \\
COE-COE-OA & 109.0 & 109.0 & 900
\end{tabular}

Both $\epsilon$ and $\sigma$ are adjusted until the CG MD simulations satisfactorily reproduce the experimental bulk density and surface tension, respectively. While the calculation of density is straightforward, for the surface tension the calculation needs some explanation. We first constructed a slab with 144 CG PEE chains in an NVT ensemble with a longer box length in the $z$-direction (nearly 10 times that of $x$ - and $y$-directions). This setup yields two air-hydrocarbon and two hydrocarbon-water interfaces (as shown in Figure 4). ${ }^{57-59}$ The total tension of this system can be written as,

$$
\tau_{\mathrm{tot}}=2\left[\gamma_{\mathrm{air}-\mathrm{hc}}+\gamma_{\mathrm{w}-\mathrm{hc}}\right]
$$

In the above equation, we use $\gamma_{\text {air-hc }}=23.37 \mathrm{dyn} / \mathrm{cm}$ and $\gamma_{\mathrm{w}-\mathrm{hc}}$ $=51.7 .{ }^{53}$ By following Lipowsky et al. ${ }^{44}$ we have calculated the total tension of the system according to the following formula,

$$
\tau=L_{z} / 2\left[P_{z z}-\left(P_{x x}+P_{y y}\right) / 2\right]
$$


TABLE 3: Parameters for Nonbonded Potentials between Various CG Sites for a System of OE7 Diblock Copolymers in Water

\begin{tabular}{lccc}
\hline \multicolumn{1}{c}{ sites } & type & $\sigma(\AA)$ & $\epsilon(\mathrm{K})$ \\
\hline W-W & LJ64 & 4.58 & 21.1 \\
W-CET & LJ96 & 5.03 & 188.0 \\
W-CEM & LJ96 & 4.89 & 193.0 \\
W-CEI & LJ96 & 5.49 & 188.0 \\
CET-CET & LJ96 & 5.48 & 138.0 \\
CET-CEM & LJ96 & 5.20 & 176.0 \\
CET-CEI & LJ96 & 5.36 & 142.0 \\
CET-COE & LJ96 & 5.08 & 88.00 \\
CET-OA & LJ96 & 4.26 & 18.0 \\
CEM-CEM & LJ06 & 4.90 & 170.0 \\
CEM-CEI & LJ96 & 5.36 & 148.0 \\
CEM-COE & LJ96 & 5.02 & 90.0 \\
CEM-OA & LJ96 & 4.52 & 18.0 \\
CEI-CEI & LJ96 & 5.50 & 102.0 \\
CEI-COE & LJ96 & 5.14 & 54.0 \\
CEI-OA & LJ96 & 5.04 & 12.0
\end{tabular}

where $L_{z}$ is the box length along the $z$-direction and $P_{i j}$ is the $i j$ component of the pressure tensors in the $i j$ direction. The prefactor, $1 / 2$ in the above equation, is appearing since there exists two interfaces. $\sigma$ is adjusted until simulations yielded a tension $(\tau)$, such that $\left|\tau_{\text {tot }}-\tau\right|<2 \mathrm{dyn} / \mathrm{cm}$. All the nonbonded parameters, thus obtained, for the hydrophobic part are listed in Table 3.

2.2. Water. We model water as a spherically symmetric interaction site (W), which represents a loose grouping of three water molecules. W sites interact via a Lennard-Jones (6-4) potential given by,

$$
U\left(r_{i j}\right)=(15 / 4) \epsilon\left[\left(\sigma / r_{i j}\right)^{6}-\left(\sigma / r_{i j}\right)^{4}\right]
$$

The quantities $\epsilon$ and $\sigma$ have a similar meaning as above. More details on the CG water model can be found elsewhere. ${ }^{45}$

2.3. Hydrophilic PEO. The interaction potential parameters among and between all the CG units for the hydrophilic part are presented in Table 3. We have developed tabulated potentials for poly (ethylene oxide) in our earlier study. ${ }^{45}$ Hence, we omit the details of treating the CG PEO part and present all the interaction potentials among and between hydrophilic groups in Figure 5.

2.4. The PEE-PEO Interface Region. Having parametrized both hydrophobic and hydrophilic parts separately, now we turn our attention to the hydrophobic-hydrophilic (PEO-PEE) interface region. This is crucial, since the diblock interface plays a major role in determining both the structure and dynamics of polymersomes. ${ }^{17}$ The PEO-PEE interface region is parametrized as before, but with two differences: (a) one additional CG unit, namely CEI, exists in this case and (b) since this is not a one-component system, the nonbonded parameters cannot be obtained in a straightforward manner as in the case of PEE. To this end, both the bond distance and bond angle parameters are obtained by comparing AA and CG bond distance and bond angle distributions, respectively. However, for the quantities involving CEI, agreement between AA and CG results is not very good. This can be rationalized, in part, by the observation that in a system with $N$ polymer chains, each with $m$ monomer units, there exist only $N$ interfacial sites in comparison to $(m-$ 1) $\times N \mathrm{EO}$ and EE sites. Hence, there is a potential problem with statistics. We tune the nonbonded parameters carefully, so as to obtain the correct interfacial tension, which is a central quantity in determining the polymersome properties. As mentioned earlier, this is not a one-component system. Hence, we choose the AA radial distribution function as a reference to
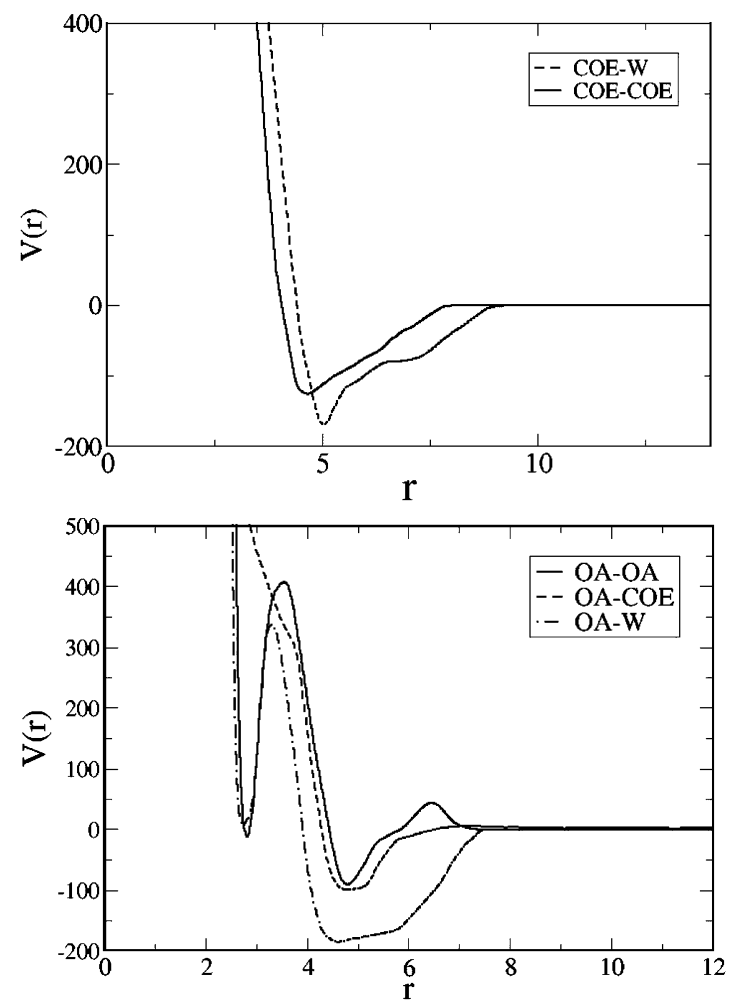

Figure 5. Tabulated potentials used among the hydrophilic (PEO block) CG units, namely COE, OA, and $\mathrm{W}$ are shown.

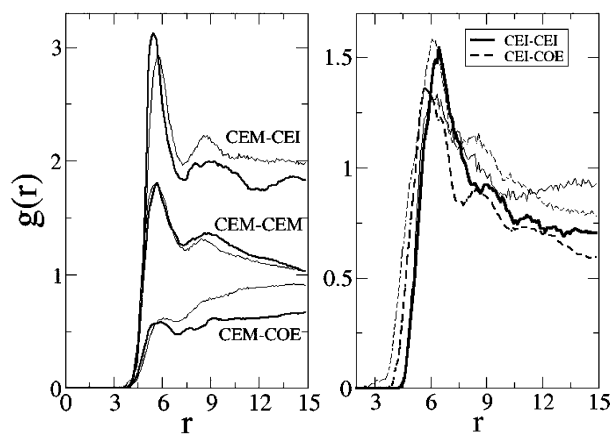

Figure 6. The radial distribution functions of various $\mathrm{CG}$ units obtained from AA (thick line) and CG (thin line) are compared. In the right panel thick solid and broken lines correspond to AA radial distribution functions, while the thin solid and broken lines represent respective CG results.

obtain the nonbonded parameters. To begin with, $\sigma$ and the potential well-depth, $\epsilon$, are adjusted so as to approximately reproduce the first peak position and height of the corresponding $g(r)$, respectively. The parameters are tuned appropriately and used as input for the next CG simulation iteratively. The iterative process continued until the CG simulations satisfactorily reproduced the AA radial distribution functions. ${ }^{60}$

In Figure 6, comparison between radial distribution functions (RDFs) obtained from AA and CG MD simulations is shown. All-atom RDFs are obtained by calculating $g(r)$ between the center of mass of the corresponding monomer units (shown in Figure 1). As can be seen from the figures, agreement between RDFs obtained from AA and CG is reasonably good. However, small discrepancies persist which can be attributed to the large CG units used in this study. All the nonbonded interaction parameter values between various CG sites are presented in Table 3. 


\section{Simulation Details}

A CG diblock copolymer chain $\left(\mathrm{EO}_{40} \mathrm{EE}_{37}\right)$ was constructed by using the $\mathrm{CG}$ parameters developed in the foregoing section. Due to the CG representation, each polymer has $78 \mathrm{CG}$ units (including a terminal tert-butyl group). The simulation system is constructed in a box by replicating a basic unit containing 4 polymer chains (two in the upper leaflet and two in the lower leaflet) and $340 \mathrm{CG}$ water sites. Polymer chains are placed in such a way that the hydrophobic tails face each other, while the hydrophilic parts are exposed to water. This system is allowed to relax and equilibrate at $298.15 \mathrm{~K}$. We then replicated the simulation cell in the $x y$-plane to generate polymer systems with respectively $8,16,32,64$, and 128 polymers. After delicate adjustments to remove overlapping water molecules, the final simulation system contained 128 copolymers and $10856 \mathrm{CG}$ water (W) sites.

The simulation system was allowed to relax and equilibrate at $298.15 \mathrm{~K}$ for $5 \mathrm{~ns}$. For the next $5 \mathrm{~ns}$, the stability of the bilayer was monitored. As mentioned in earlier studies, ${ }^{61}$ the $\mathrm{CG}$ approach involves larger size interacting sites and softer potentials, which essentially results in the time scales that are dilated by 2 orders of magnitude larger in comparison to the all-atom simulation times. All of the times reported in this article are the actual CG simulation times, and not the effective simulation times that are likely 2 orders of magnitude longer.

3.1. Constant Area Simulations. To compare our simulation results with the experimental results, it is necessary to carry out MD simulations with constant surface area for each polymer $\left(P_{\mathrm{A}}\right)$ that corresponds to zero tension. This is an important observation because the surface/interfacial tension of the system, which is very sensitive to the quantity $P_{\mathrm{A}}$, can vary over an order of magnitude by moderately changing surface area. ${ }^{17}$ Hence, for this purpose we have chosen an NAPT ensemble. ${ }^{43,44,57}$ In this ensemble, the simulation box length in the $x$ - and $y$-directions is fixed while it is allowed to vary in the $z$-direction. As a result, the normal pressure in the $z$-direction fluctuates around a mean (pressure) value, while keeping the surface area per polymer unchanged. Such methodology was used earlier by Lipowsky et al. ${ }^{43,44}$ to determine the interfacial tension of self-assembled model bilayers.

3.2. The Zero-Tension System. The simulation system constructed with a default $P_{\mathrm{A}}$ yielded an arbitrary value of $\tau$. Since the experiments are generally carried out at zero tension, it is important to determine the surface area per polymer corresponding to zero tension. This task is accomplished in the following manner. The initial system having area per polymer $P_{\mathrm{A}}=113.1 \AA^{2}$ resulted in $\tau=18.5 \mathrm{dyn} / \mathrm{cm}$. This system was then studied in both expanded (increased tension) and compressed (decreased tension) states by dilating the $x y$-plane by approximately 5\% along $x$ and $y$. The resulting systems with different fixed surface area are allowed to relax and then equilibrated for $5 \mathrm{~ns}$. The total tension of each system was then determined according to eq 5 . The variation in tension with surface area is plotted in Figure 7. Symbols show the simulation result while the full line is a linear fit. The dashed horizontal line corresponds to zero tension. From the intersection of these two straight lines, we determine the area per polymer that corresponds to zero tension. In this case we find $\mathrm{P}_{\mathrm{A}}{ }^{o}=104.2$ $\AA^{2}$. A simulation cell with this area per polymer was constructed and the simulation results thus obtained were compared with experiments (see below).

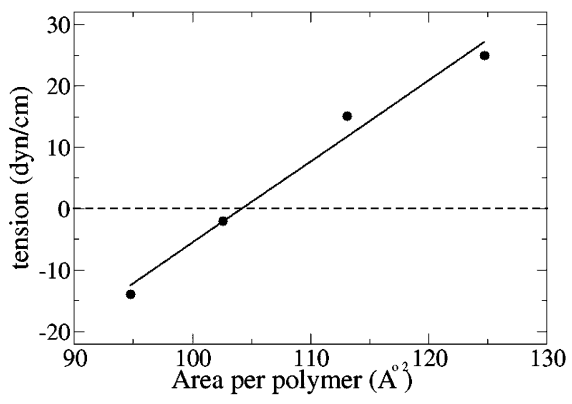

Figure 7. The total tension, $\tau$, of the polymersome membrane is plotted as a function of polymer surface area, $P_{\mathrm{A}}$. The dots are the CG simulation results, while the full line is a linear fit to the data. The dashed horizontal line corresponds to $\tau=0 . P_{\mathrm{A}}{ }^{o}$ (area per polymer at $\tau=0$ ) is determined from the intersection of the two straight lines.

\section{Results}

4.1. Polymersome Properties. A snapshot taken from a simulation of a $\mathrm{EO}_{40} \mathrm{EE}_{37}$ bilayer is shown in Figure 8. The thick yellow portion corresponds to the EE hydrophobic core, red chains to the PEO and light blue spheres represent $\mathrm{W}$ particles, the CG water. In the same figure mass density profiles for the components of the bilayer at zero tension are shown as a function of distance from the bilayer center. Close inspection of the density profiles reveals that the water penetrates the PEO-PEE interfacial region. A recent experimental study ${ }^{62}$ also showed that the water swells PEO chains at the PEO-PEE interface, which in turn prevents the possible PEO phase transition, thereby making the vesicles more stable. The hydrophobic thickness calculated from density profiles is 7.68 $\mathrm{nm}$, which is in good agreement with the experimental observations $(\sim 8 \pm 1 \mathrm{~nm})$.

4.2. Self-Assembly. The results presented in the previous section were obtained by carrying out simulations on a preassembled zero-tension bilayer. Here, we study the self-assembly of block copolymers. For this purpose, we have selected relatively smaller copolymers compared to OE7, namely $\mathrm{EO}_{10^{-}}$ $\mathrm{EE}_{9}$ diblock copolymers. Note that this copolymer continues to have $\sim 38 \%$ hydrophilic fraction (similar to that of OE7), which is a typical lipid-like hydrophobic/hydrophilic ratio.

The simulation system was constructed by initially distributing CG polymer chains on a 3D cubic lattice and hydrating the resulting system with sufficient $\mathrm{CG}$ water sites. The resulting simulation system contains $108 \mathrm{EO}_{10} \mathrm{EE}_{9}$ copolymers and 2160 $\mathrm{W}$ particles. This system was then first equilibrated at the desired thermodynamic state (298.15 K temperature and $1 \mathrm{~atm}$ pressure) by keeping the polymers immobile. This results in a suitable initial unbiased lattice configuration. Since we aim to study natural self-assembly, to avoid unwanted compressions/expansions during the simulations, an isobaric NPT ensemble was used. This ensemble isotropically compresses/expands the box lengths, in contrast to the general NPT ensemble.

Starting from the initial lattice configuration, the dynamics of spontaneous self-assembly was monitored. The initial configuration and the snapshots obtained during the self-assembly are presented in Figure 9. The copolymers start self-assembling spontaneously through the association of hydrophobic groups, which cluster together. The cluster size increases with time. Hydrophilic segments, being relatively smaller, are unable to prevent PEE chains from being exposed to water. This likely motivates the rapid aggregation among the hydrophobic segments in a fashion that minimizes the exposure of hydrophobic segments to water. The clusters subsequently readjust to protect hydrophobic segments from water exposure. Self-assembly in this case results in a bilayer (as shown in Figure 9). 

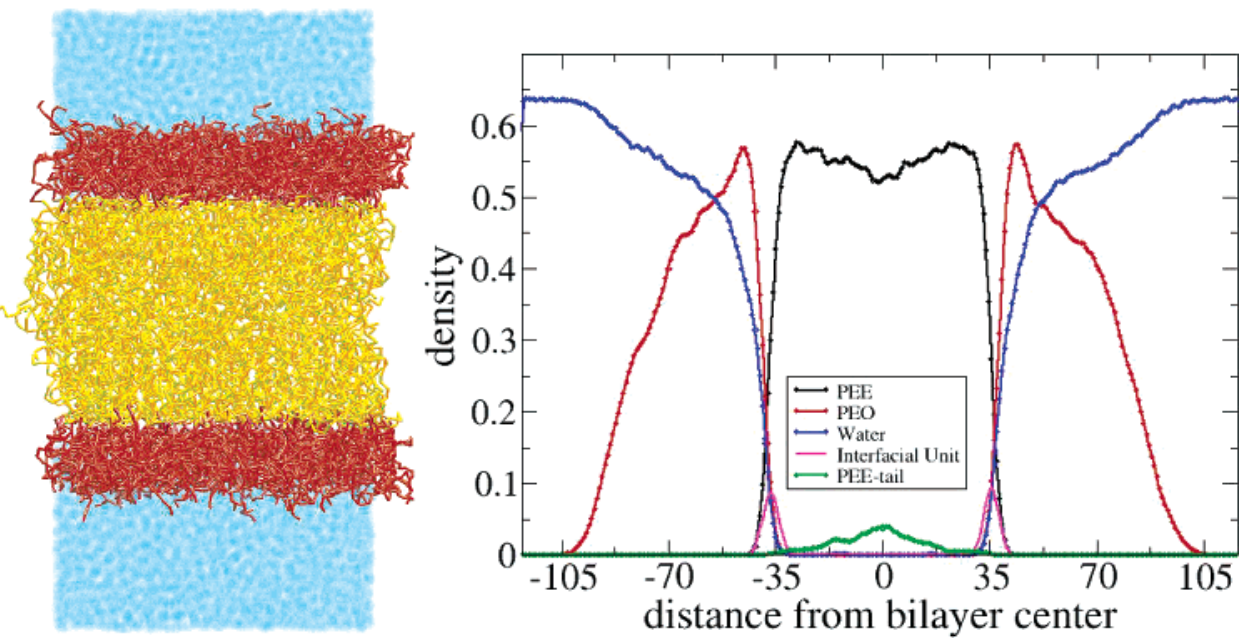

Figure 8. Snapshot taken from a CG simulation on a preassembled OE7 bilayer. The right panel shows density profiles for individual components of the bilayer. The color code is the following: PEE, yellow; PEO, red; and water, blue.
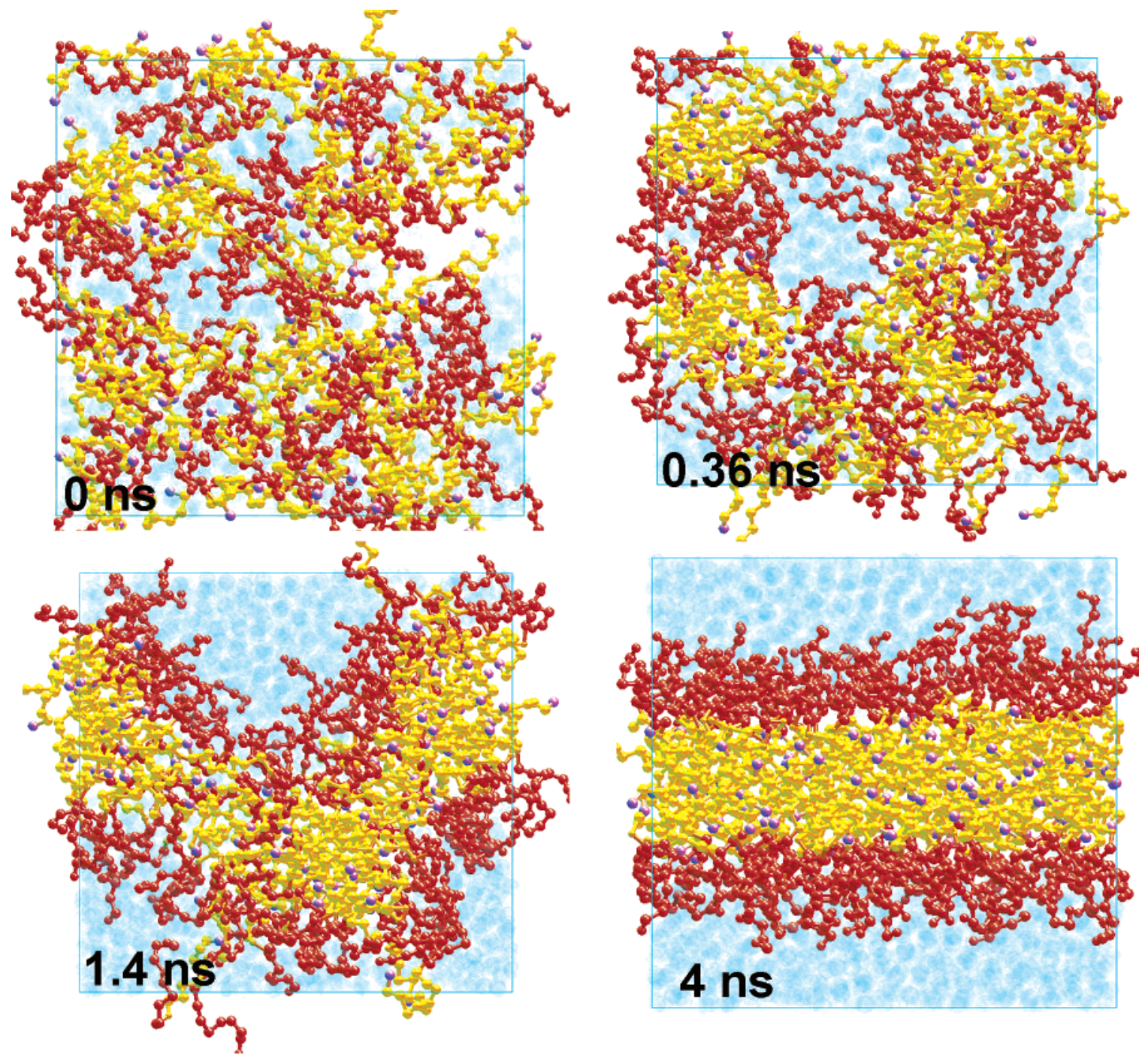

Figure 9. Self-assembly of diblock copolymers as observed in CG simulations. The simulation started from a lattice configuration with 108 $\mathrm{EO}_{10} \mathrm{EE}_{9}$ diblock copolymers in $2160 \mathrm{CG}$ water sites (the color code is the same as in Figure 8). Hydrophobic tail ends are shown in violet.

To understand the hydrophilic dependence of the block copolymers, it is necessary to study the self-assembly of copolymers by varying the hydrophilic fraction. For this purpose we have chosen $\mathrm{EO}_{19} \mathrm{EE}_{9}$ polymer chains corresponding to the $60 \%$ hydrophilic fraction. In this case the simulation system is constructed by placing 48 copolymers among 6912 CG water molecules as before. Note that as more water is added, the hydrophobic block increasingly avoids hydration, which results in an increase in the interfacial tension tending to straighten the interface and increase the hydrophobic thickness. ${ }^{63}$ Starting 

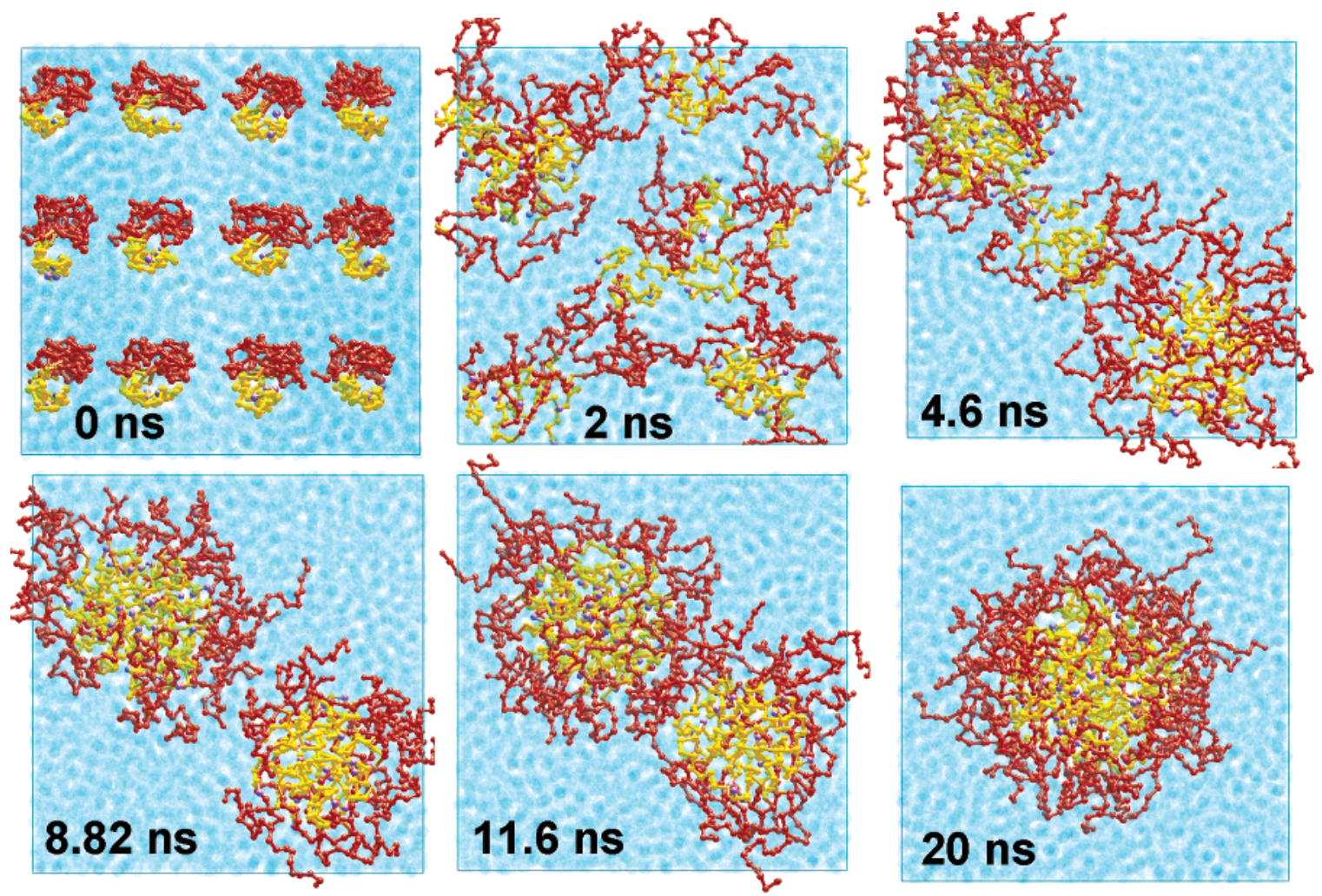

Figure 10. Self-assembly of diblock copolymers with a larger hydrophilic fraction (60\%) as observed in CG simulations. The simulation started from a lattice configuration with $48 \mathrm{EO}_{19} \mathrm{EE}_{9}$ diblock copolymers among $6912 \mathrm{CG}$ water sites (the color code is the same as in Figure 9). The self-assembly in this case resulted in a spherical micelle as shown in the figure.

from a lattice configuration, spontaneous self-assembly of $\mathrm{EO}_{19^{-}}$ $\mathrm{EE}_{9}$ copolymers is studied. Corresponding simulation snapshots are shown in Figure 10. Copolymers start self-assembling spontaneously through the hydrophobic groups gathering together. The self-assembly study in this case revealed similar features to that of bilayer formation. The initial lattice configuration starts forming local aggregates which diffuse gradually and reorient themselves in a way to prevent the hydrophobic segments from water exposure. The presence of a large hydrophilic fraction makes this task easier and hence the spontaneous self-assembly results in spherical micelle formation as shown in Figure 10. The resulting spherical micelle is observed to be stable until the end of the simulation (20 ns).

The simulation results described above can be interpreted in a straightforward manner. The unique driving force behind the spontaneous self-assembly in this case is the hydrophobic interaction of PEE segments with water. Hence the copolymers try to adopt a structure in which the PEE chains are minimally exposed to water. In other words, the surface area of the final configuration should be able to be completely covered by PEO chains to the maximum extent. An obvious choice is a spherical micelle, where the hydrophobic blocks are completely hidden inside, while the PEO chains form an outer corona. As expected, this is observed in the case of $\mathrm{EO}_{19} \mathrm{EE}_{9}$, where the hydrophilic segments are long enough to cover the hydrophobic core.

\section{Conclusions}

In this work, we have developed a coarse-grain model for polymersome membranes. The simplicity of the CG method allows us to probe polymersome self-assembly as it occurs locally on the nanosecond to microsecond time scale. Starting from a random configuration, we showed that suitable block copolymers with a lipid-like hydrophilic fraction spontaneously self-assemble into a bilayer while the larger hydrophilic fraction facilitates the spherical micelle formation.

The results obtained from CG simulations are in good agreement with experimental observations. For example, the hydrophobic thickness of the OE7 bilayer agrees very well with the experimental findings. The density profile study suggested that the water penetrates the hydrophobic-hydrophilic interface region to a considerable extent. This is in accordance with a recent experimental study, concluding that the water swells PEO chains.

As mentioned earlier, the average shape of the molecule is most simply a reflection of the hydrophilic-hydrophobic ratio. In this work we have studied self-assembly with a lipid-like hydrophilic/hydrophobic ratio $\left(f_{\text {hydrophilic }}=38 \%\right)$. In an effort to understand the $f_{\text {hydrophilic }}$ dependence of the microstructures, we have studied the self-assembly of copolymers by varying the hydrophilic fraction. Block copolymers with $60 \%$ hydrophilic fraction self-assembled into a stable spherical micelle configuration.

Experimental studies revealed that the OE7 vesicles show nearly $10 \%$ more polysdispersity compared to that of natural biomembranes. In the present study we have not taken this fact into account. All the systems we have studied are monodisperse systems. However, experimental studies ${ }^{16,17,31}$ concluded that the results in this polydisperse system are not affected by the small population present at the ends of the polydispersity distribution. To examine this point and also to see to what extent the present results are affected by polydispersity, it is also necessary to study a polydisperse system. Work in this direction is under way. ${ }^{64}$

Block copolymers containing PLA (polylactic acid) are of considerable interest due to the hydrolytic biodegradation of PLA, which is widely exploited in controlled drug release. ${ }^{65,66}$ Water-soluble block copolymer micelles have been tested as 
hydrophobic drug carriers, ${ }^{66-69}$ but PEO-PLA vesicles are only now being reported. ${ }^{17}$ The present study promises the feasibility of modeling such systems. We likewise aim to develop such models with suitable CG potentials and perhaps study chemical degradability in our future work.

Acknowledgment. We thank Carlos Lopez and Preston Moore for their interest. This research was supported by the National Institute of Health and the National Science Foundation.

\section{References and Notes}

(1) Lipowsky, R.; Sackmann, E., Eds. Structure and dynamics of membranes; Elsevier Science: Amsterdam, The Netherlands, 1995. 1993.

(2) Cevc, G. Phospholipids handbook; Marcel Decker: New York,

(3) Nagle, J. F.; Nagle, S. T. Biochim. Biophys. Acta 2000, 14, 159.

(4) Tobias, D. J.; Tu, K.; Klein, M. L. Curr. Opin. Struct. Biol. 1997,

2, 15-26. Saiz, L.; Klein, M. L. Acc. Chem. Res. 2002, 35, 482.

(5) Lopez, C. F.; Montal, N.; Blasie, J. K.; Klein, M. L.; Moore, P. B. Biophys. J. 2002, 83, 1259 2484.

(6) Moore, P. B.; Lopez, C. F.; Klein, M. L. Biophys. J. 2001, 81,

(7) Scott H. L. Curr. Opin. Struct. Biol. 2002, 12, 495.

(8) Balasubramanian, S.; Bagchi, B. J. Phys. Chem. B 2002, 106, 3668.

Balasubramanian, S.; Pal, S.; Bagchi, B. Phys. Rev. Lett. 2002, 89, 115505.

(9) Tew, G. N.; Liu, D.; Chen, B.; Doerksen, R.; Kaplan, J.; Carroll;

Klein, M. L.; DeGrado, W. F. Proc. Natl. Acad. Sci. U.S.A. 2002, 99, 5110

(10) O’Brien, D. F.; Klingbiel, R. T.; Specht, D. P.; Tyminski, P. N. Ann. N.Y. Acad. Sci. 1985, 446, 282.

(11) van Hest, J. C. M.; Delnoye, D. A. P.; Baars, M. W. P. L.; van

Genderen, M. H. P.; Meijer, E. W. Science 1995, 268, 1592.

(12) Zhou, S. Q. et al Science 2001, 291, 1944.

(13) Jung, H. T.; Coldren, B.; Zasadzinski, J. A.; Iampietro, D. J.; Kaler,

E. W. Proc. Natl. Acad. Sci. U.S.A. 2001, 98, 1353.

(14) Cornelissen, J. J. L.; Fischer, M.; Sommerdijk, N. A. J. M.; Nolte,

R. J. M. Science 1998, 280, 1427.

(15) Hillmayer, M.; Bates, F. S. Macromolecules 1996, 29, 6994.

(16) Discher, B. M.; Won, Y. Y.; Ege, D. S.; Lee, J. C.-M.; Bates, F.

S.; Discher, D. E.; Hammer, D. A. Science 1999, 284, 1143.

(17) Disher, D. E.; Eisenberg, A. Science 2002, 297, 967.

(18) Hamley, I. W. Nanotechnology 2003, 14, R39.

(19) Dimova, R.; Seifert, U.; Poligny, B.; Forster, S.; Dobereiner, H. G. Eur. Phys. J. E 2002, 7, 241.

(20) Meier, W.; Nardin, C.; Winterhalter, M. Angew. Chem., Int. Ed. 2000, 39, 4599 .

(21) Nardin, C.; Hirt, T.; Leukel, J.; Meier, W. Langmuir 2000, 16, 1035.

(22) Balsara, N. P.; Garetz, B. A.; Newstein, M. C.; Bauer, B. J.; Prosa,

T. J. Macromolecules 1998, 31, 7668.

(23) Bermudez, H.; Brannan, A. K.; Hammer, D. A.; Bates, F. S.; Discher, D. E. Macromolecules 2002, 35, 8203.

(24) Forster, S.; Zisenis, M.; Wenz, E.; Antonietti, M. J. Chem. Phys. 1996, 104, 9956.

(25) Zhang, L.; Eisenberg, A. Science 1995, 268, 727.

(26) Schillen, K.; Bryskhe, K.; Mel'nikova, Y. S. Macromolecules 1999, 32,6885 .

(27) Discher, B. M.; Won, Y. Y.; Ege, D. S.; Lee, J. C-M.; Bates, F. S.; Discher, D. E.; Hammer, D. A. Science 1999, 284, 1143.

(28) Needham, D.; Zhelev, D. In The mechanochemistry of lipid vesicles examined by micropipette manipulation techniques in vesicles; Rosoff, M., Ed.; Marcel Dekker: New York, 2000; Chapter 9.

(29) Rawicz, W.; Olbich, K. C.; McIntosh, T.; Needham, D.; Evans, E. Biophys. J. 2000, 79, 328.

(30) Luo, L.; Eisenbeerg, A. J. Am. Chem. Soc. 2001, 123, 1012.

(31) Aranda-Espinoza, H.; Bermudez, H.; Bates, F. S.; Discher, D. E. Phys. Rev. Lett. 2001, 87, 208301.

(32) Israelachvili, J. Intermolecular and surface forces, 2nd ed.; Academic Press: New York, 1991.

(33) Fraaije, J. G. E.; Sevink, G. J. A. Macromolecules 2003, 36, 7891.

(34) Morozov, A. N.; Fraaije, J. G. E. Macromolecules 2001, 34, 1526.

(35) Malfreyt, P.; Tildesley, D. J. Langmuir 2000, 4372.
(36) Groot, R. D.; Madden, T. J.; Tidesley, D. J. J. Chem. Phys. 1999 , 110, 9739. Groot, R. D.; Rabone, K. L. Biophys. J. 2001, 81, 725. Groot, R. D. J. Chem. Phys. 2003, 118, 11265.

(37) Leibler, L. Macromolecules 1980, 13, 1602. Pakula, T.; Karatasos, T.; Anastasiadis, S. H.; Fytes, G. Macromolecules 1997, 30, 8463.

(38) Shillcock, J. C.; Lipowsky, R. Chem. Phys. 2002, 117, 5048.

(39) Schultz, A. J.; Hall, C. K.; Genzer, J. J. Chem. Phys. 2002, 117, 10329

(40) Fried, H.; Binder, K. J. Chem. Phys. 1991, 94, 834

(41) Pastor, R.; Venable, R. M.; Karplus, M.; Szabo, A. J. Chem. Phys. 1988, 89,1128

(42) Nagochi, H. J. Chem. Phys. 2002, 117, 8130. Nagochi, H.; Takasu, M. J. Chem. Phys. 2001, 115, 9547. 221

(43) Goetz, R.; Gompper, G.; Lipowsky, R. Phys. Rev. Lett. 1999, 82,

(44) Goetz, R.; Lipowsky, R. J. Chem. Phys. 1998, 108, 7397.

(45) Shelley, J. C.; Shelley, M. Y.; Reeder, R. C.; Bandyopadhyay, S.; Klein, M. L. J. Phys. Chem. B 2001, 105, 4464. Shelley, J. C.; Shelley, M. Y.; Reeder, R. C.; Bandyopadhyay, S.; Moore, P. B.; Klein, M. L. J. Phys. Chem. B 2001, 105, 9785.

(46) Nielsen, S.; Klein, M. L. In Bridging time scales: Molecular simulations for the next decade; Nielaba, P., Mareschali, M., Ciccotti, G., Eds.; Springer-Verlag: Berlin, Germany, 2002; pp 25-63.

(47) Nielsen, S.; Lopez, C. F.; Srinivas, G.; Klein, M. L. J. Chem. Phys. 2003, 119, 7043. Lopez, C. F.; Nielsen, S.; Moore P. B.; Klein, M. L. Proc. Natl. Acad. Sci. U.S.A. 2004, 101, 4431. Nielsen, S. O.; Lopez, C. F. Srinivas, G.; Klein, M. L. J. Phys. Condens. Matter 2004, 16, 481.

(48) De Vries, A. H.; Mark, A. E.; Marrink, S. J. J. Am. Chem. Soc. 2004, 126, 4488

(49) Tieleman, D. P.; Leontiadou, H.; Mark, A. E.; Marrink, S. J. J. Am. Chem. Soc. 2003, 125, 6382. Marrink, S. J.; Lindahl, E.; Edholm, E. Mark, A. E. J. Am. Chem. Soc. 2001, 123, 8638. Marrink, S. J.; Mark, A. E. J. Am. Chem. Soc 2003, 125, 11144.

(50) Marrink, S. J.; Mark, de Vries, A. H.; Mark, A. E. J. Phys. Chem. $B$ 2004, 108, 750 .

(51) Smit, B.; Hilbers, P. A. J.; Esselink, K.; Rupert, L. A. M.; Van Os, N. M.; Schlijper, A. G. J. Phys. Chem. 1991, 95, 6361. Kranenburg, M. Venturoli, M.; Smit, B. J. Phys. Chem. B 2003, 107, 11491.

(52) Venturoli, M.; Smit, B. PhysChemComm 1999, 10.

(53) Physical properties of polymer handbook; Mark, J. E. Ed.; American Institute of Physics: Woodbury, NY, 1996.

(54) Hamley, I. The Physics of Block Copolymers; Oxford University Press: New York, 1998.

(55) Damm, W.; Frontera, A.; Tirado-Rives, J.; Jorgensen, W. L. Comput. Chem. 1997, 18, 1955.

(56) Jorgensen, W. L.; Maxwell, D. S.; Tirado-Rives, J. J. Am. Chem Soc. 1996, 118, 11225 .

(57) Feller, S. E.; Pastor, R. W. J. Chem. Phys. 1999, 111, 1281.

(58) Marrink, S. J.; Mark, A. E. J. Phys. Chem. B 2001, 105, 6122

(59) Lindahl, E.; Edholm, E. J. Chem. Phys. 2000, 113, 3882.

(60) Lyubarstev, A. P.; Laaksonen, A. Phys. Rev. E 1995, 52, 3730.

(61) Lopez, C. F.; Moore, P. B.; Shelley, J. C.; Shelley, M. Y.; Klein,

M. L. Comput. Phys. Commun. 2002, 147, 1.

(62) Won, Y.-Y.; Davis, H. T.; Bates, F. S.; Agmalian, M.; Wignall, G. D. J. Phys. Chem. B 2000, 104, 7134 .

(63) The single-walled or unilamellar vesicles undergo a reversible change in thickness from 45 to $100 \mathrm{~nm}$ as water content is changed from $25 \%$ to $75 \%$. Such findings motivated us to stick to a particular copolymer/ water ratio, based on the hydrophilic fraction. Note that as more water is added, the hydrophobic block increasingly avoids hydration. This results in an increase in the interfacial tension, $\gamma$, which tends to straighten the interface and increase the hydrophobic thickness.

(64) Ortiz, V.; Srinivas, G.; Discher, D. E.; Klein, M. L. Manuscript in perperation.

(65) Hagan, S. A.; Coombes, A. G. A.; Garnett, M. C.; Dunn, S. E.; Davies, M. C.; Illum, L.; Davis, S. S.; Harding, S. E.; Purkiss, S.; Gellert, P. R. Langmuir 1996, 12, 2153.

(66) Stolnik, S.; Illum, L.; Davis, S. S. Adv. Drug Delivery Rev. 1995 $16,195-214$

(67) Savic, R.; Luo, L.; Eisenberg, A.; Maysinger, D. Science 2003, $300,615-618$

(68) Ding, J.; Liu, G. J. Phys. Chem. B 1998, 102, 6107-6113.

(69) Nardin, C.; Widmer, J.; Winterhalter, M.; Meier, W. Eur. Phys. J. E 2001, 4, 403-410. 